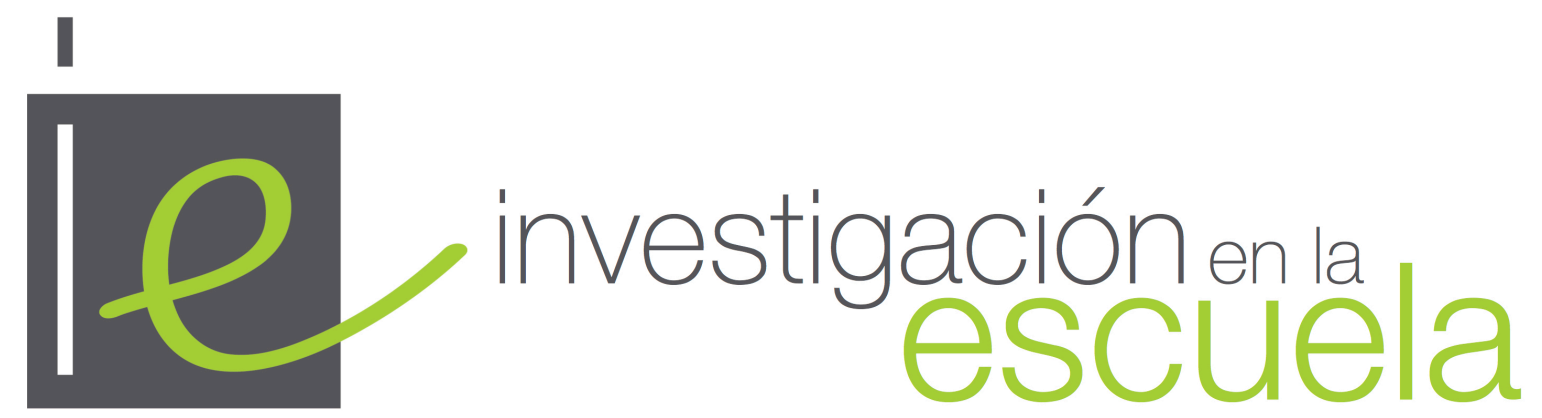

Revista internacional de investigación e innovación educativa

\title{
La Historia como formadora de ciudadanía: concepciones de estudiantes de Bachillerato de un centro de Sevilla
}

\author{
José Cano Romero y Elisa Navarro-Medina \\ Universidad de Sevilla \\ España
}

Citación: Cano Romero, J. y Navarro-Medina, E. (2019). La Historia como formadora de ciudadanía: concepciones de estudiantes de Bachillerato de un centro de Sevilla. Investigación en la Escuela, 99, 60-74. doi: http://dx.doi.org/10.12795/IE.2019.199.05

Resumen: El objetivo de esta investigación ha sido analizar si los estudiantes son capaces de posicionarse críticamente con respecto a ciertas problemáticas sociopolíticas, si pueden establecer relaciones de causalidad entre fenómenos actuales y acontecimientos pasados y si valoran la utilidad de la Historia en relación al ejercicio de su papel como ciudadanos de una democracia. Participaron en el estudio una muestra de 18 estudiantes de Bachillerato de un centro público de la ciudad de Sevilla (España). Se recabaron datos a partir de una entrevista estructurada, que se analizó con el programa Atlas.ti, utilizando un sistema de categorías en hipótesis de progresión de lo simple a lo complejo. Los resultados ponen de manifiesto que los estudiantes no utilizan el conocimiento histórico para explicar problemáticas de actualidad y posicionarse en torno a ellas.

Palabras clave: "Educación ciudadana"; "enseñanza secundaria"; "enseñanza de la Historia”; "educación para los derechos humanos"; "ciudadanía”.

History as a trainer of citizenship: conceptions of high school students from a centre in Seville 
Abstract: The purpose of this research was to analyse whether students are able to express a strong position on some socio-political problems, whether they can find cause-effect relationships between current historical phenomena and past events and whether they appreciate the usefulness of History in connection with their role of citizens in a democracy. A group of 18 A Level students from a state high school of Seville (Spain) took part in this study. Data were collected thanks to a structured interview, whose data were analysed with the Atlas.ti program, using a system of categories with progression hypotheses which move from simple to complex. The results show that students do not use historical knowledge to explain current problems and position themselves around them.

Key words: "Citizenship education"; "secondary education"; "history education"; "human rights education"; "citizenship".

Histoire comme formateur de citoyenneté: Concepts de lycéens dans un centre à Séville Resumè: L'objectif de cette recherche a été d'analyser si les élèves sont capables de se positionner de manière critique par rapport à certains problèmes sociopolitiques, s'ils peuvent établir des relations de cause à effet entre les phénomènes actuels et les événements passés, et s'ils valorisent l'utilité de l'histoire par rapport à l'exercice de leur rôle de citoyens d'une démocratie. Un échantillon de 18 élèves du secondaire d'une école publique de la ville de Séville (Espagne) a participé à l'étude. Les données ont été collectées à partir d'une interview structurée, qui a été analysée avec le programme Atlas.ti, en utilisant un système de catégories dans l'hypothèse d'une progression du simple au complexe. Les résultats montrent que les élèves n'utilisent pas les connaissances historiques pour expliquer les problèmes actuels et se positionner par rapport à ceux-ci.

Mots-clés: "Éducation à la citoyenneté"; "enseignement secondaire"; "enseignement de l'histoire"; "éducation aux droits de l'homme"; "citoyenneté".

\section{Introducción}

La formación ciudadana del alumnado, por un lado, y el aprendizaje y la enseñanza de las materias de Ciencias Sociales -entre las que se halla Historia de España de Segundo de Bachilleratoguardan una estrecha y necesaria relación. Ser partícipes de un sistema político democrático y ejercer como ciudadanos activos y críticos dentro de éste requiere, cuanto menos, un conocimiento del pasado más reciente que permita a dicho ciudadano establecer relaciones entre la Historia y su presente más inmediato.

Sin embargo, esta relación no puede darse sin poner de manifiesto los fines del sistema educativo en el que debe de desarrollarse. En este sentido, son muchos los autores que señalan la predominancia de un paradigma en el que gran parte de las concepciones de la educación que se manejan en el mundo tienden a privilegiar el fin mercantilista de ésta (De Alba y García Pérez, 2008; Durán, 2012; García Pérez, 2012, 2016; Gimeno, 2010; Giroux, 2015; Padilla, 2014). Ello implica que el sistema educativo actual esté fomentando la llamada "cultura de la superficialidad" (García Pérez, 2012, p. 268) y el "pensamiento dominante" (De Alba y García Pérez, 2008, p. 7), dado que "lo que más importa es que los educandos logren las competencias valiosas para el ingreso en el mundo del trabajo y el mantenimiento de la competitividad de las economías” (Gimeno, 2010, p. 238).

Pero a nuestro juicio, la función del sistema educativo debería de ser otra bien distinta, basada en que los estudiantes afronten "los desafíos actuales de todo tipo: la violencia, la globalización, la tendencia al pensamiento único, el fenómeno de la interculturalidad o los grandes desequilibrios que sufren las sociedades actuales" (Zaitegi, 2004, p. 708). Y para ello no sólo es necesario prepararlos laboralmente, sino y sobre todo socialmente, requiriendo "del individuo 
conocimientos, habilidades y valores para lograr su compromiso y responsabilidad ante dilemas de la sociedad" (Zambrano, 2018, p. 70).

Por tanto, ¿cuál debería ser el objetivo último de un sistema educativo en democracia? En resumidas cuentas, podríamos decir que éste no es otro que "fomentar en los alumnos su capacidad de participación en la vida social en un sistema democrático, es decir, en un sistema en el que existe igualdad de derechos y de deberes para todos con independencia de su posición social o de sus creencias" (Delval, 2012, p. 43). Y para ello es necesario democratizar la propia institución escuela (Moliner, Traver, Ruiz y Segarra, 2016, p. 118).

\section{Una visión alternativa de la enseñanza y el aprendizaje de la Historia}

Sin embargo, ni estos fines educativos, ni la capacidad de la Historia como formadora de ciudadanía parece que estén calando en los estudiantes ni en el modelo de ciudadano que se pretende (Navarro-Medina y De-Alba-Fernández, 2018). Aunque podemos encontrar numerosas razones, hay dos que tienen una fuerza especial: el currículo oficial presente en la actual legislación educativa basado en un listado de temas correlativos, principalmente políticos, y descontextualizados del presente- y la Prueba de Evaluación del Bachillerato para el Acceso a la Universidad al final de la citada etapa -de carácter eminentemente memorística-.

Por consiguiente, ¿cómo debería aprenderse Historia? Grosso modo, sostenemos que ésta no debe enseñarse "como una relación de reyes y batallas, propia de la educación tradicional-elitista de épocas pasadas" (De-Alba-Fernández y Navarro-Medina, 2013, p. 720), sino que debería tratar de concebirse la Historia como un todo, integrando sus dimensiones económica, social y cultural. Y para ello es necesario estructurarla "en torno a problemas sociales relevantes que permitan no sólo organizar el contenido, sino favorecer las capacidades de comprensión, interpretación e inferencia de los estudiantes" (De-Alba-Fernández y Navarro-Medina, 2013, p. 725).

Además de abogar por el fomento de una organización alternativa de los contenidos, también sería preciso cuestionarnos qué contenidos se habrían de impartir y cuáles no. En este sentido, la enseñanza de la Historia debería "ayudar a aflorar, a ver la luz a grupos sociales y problemas olvidados por las grandes tendencias históricas y por no pocos manuales escolares al uso" (De la Montaña, 2015, p. 911), dado que "cuando los contenidos de historia se ocupan de asuntos desconectados de los problemas sociales que afectan a la gente de hoy, nos encontramos con la historia de anticuario, pasatiempo de minorías ociosas" (López Facal, 2002, p. 250). En este sentido, conviene trabajar una historia de tipo social, "desde abajo", que tenga en consideración la vida cotidiana de las personas y que dé visibilidad a grupos sociales tradicionalmente marginados del relato histórico frente a los grupos dominantes (De la Montaña, 2015, p. 912).

\section{Segunda República, Guerra Civil y franquismo y su relación con la memoria histórica y el proceso de enseñanza-aprendizaje de Historia de España}

En la necesidad de partir desde problemas sociales pasados para entender el presente, pocos acontecimientos históricos han marcado tanto el devenir de España como aquellos acaecidos entre los años 1931 y 1975. Por tanto, ¿qué enseñanza de la Segunda República, la Guerra Civil y el franquismo cabe esperar en una escuela que forme a ciudadanos y ciudadanas del siglo XXI?

En primer término, se hace necesario abogar por una Historia que deje de lado la denominada "teoría de la equidistancia", predominante en los manuales escolares desde la llegada de la democracia y la cual considera que ambas partes fueron igualmente responsables del desencadenamiento de la guerra (Valls, 2009, p. 64). De igual modo, se debe abandonar el olvido de 
"la represión franquista sistemática y orquestada legalmente y con carácter retroactivo que se prolongó durante los 40 años posteriores a la guerra civil” (Díez, 2013, p. 401), presente aún en muchos manuales.

Ello entronca directamente con la necesidad de que la memoria histórica, entendida ésta como el recuerdo "de los momentos traumáticos" (Delgado, 2015, p. 99), se halle presente en los currículos escolares, ya que si pretendemos educar para una ciudadanía crítica, respetuosa con los Derechos Humanos y con la democracia y partícipe de las problemáticas de la comunidad en la que habita, es más que necesario el trabajo en el aula de los temas de la Guerra Civil y la represión franquista, así como el surgimiento de una democracia en España con la Segunda República.

Sin embargo, la presencia de la memoria histórica en el ámbito escolar no solo es importante debido a las razones ya mencionadas, sino también a que es necesario hacer un uso adecuado del pasado a fin de que nos llegue a favorecer a nuestro presente (Bejines, 2016), previniendo además "la repetición o la negación de acontecimientos devastadores que marcaron el siglo XX” (Díez, 2013, p. 406).

\section{Método}

El objetivo de este estudio ha sido analizar si los estudiantes de Segundo de Bachillerato son capaces de establecer relaciones entre fenómenos de actualidad y acontecimientos del pasado, si pueden posicionarse críticamente en relación con ciertos temas de actualidad relacionados con la Historia y si valoran la utilidad de ésta en democracia.

La muestra, seleccionada por conveniencia, han sido 18 estudiantes de entre 17 y 18 años, de los cuales un $61 \%$ son mujeres, un $17 \%$ son hombres y un $22 \%$ ha preferido no decir cuál es su género o indicar que pertenece a algún otro de tipo no binario. Los estudiantes están matriculados en Segundo de Bachillerato, en la modalidad de Ciencias, de un instituto público ubicado en un barrio de clase media de la ciudad de Sevilla (España).

Como instrumento de recogida de información se ha utilizado la técnica de la entrevista estructurada. Los datos han sido recogidos en el mes de abril de 2019. Las preguntas que componen el instrumento son las siguientes:

1. Como ciudadano de un sistema democrático, puedes ejercer una serie de derechos. ¿Te ha ayudado en algo la asignatura de Historia de España en este sentido? En caso afirmativo, ¿cómo?

2. ¿Qué conocimientos de los que has aprendido durante el curso crees que te han resultado más útiles y por qué?

3. ¿Para qué crees que estudiamos Historia?

4. ¿Crees que existe alguna relación entre los períodos históricos de la Segunda República, la Guerra Civil y el franquismo -u otros momentos de la Historia de España- y los siguientes temas de actualidad?:

a) La crisis económica.

b) La incapacidad de acuerdo entre los principales grupos políticos en las dos últimas legislaturas.

c) La violencia de género.

d) El auge de VOX.

e) Los casos de corrupción política.

5. ¿Crees que a día de hoy España sigue teniendo problemas no superados que vienen de lejos? ¿Por qué? En caso afirmativo, ¿cuáles? 
6. ¿Cómo dirías que funciona la democracia en España? ¿Piensas que guarda relación con algún acontecimiento de nuestra historia?

7. ¿Piensas que, en ciertos casos, una dictadura podría ser preferible a un sistema democrático o, por el contrario, no?

Para el análisis de las respuestas, que han sido categorizadas mediante el programa Atlas.ti, hemos recurrido a un sistema de categorías emergente (Mayring, 2014) en hipótesis de progresión, de lo simple a lo complejo (García Díaz, 1995), dando como resultado tres variables con diferentes niveles de definición cada una de ellas:

Tabla 1

Descripción de las variables y sus niveles

\begin{tabular}{|c|c|c|c|}
\hline Variables & Nivel 1 & Nivel 2 & Nivel 3 \\
\hline $\begin{array}{l}\text { Variable 1: Valoración } \\
\text { de la utilidad de la } \\
\text { Historia en relación } \\
\text { con el ejercicio de la } \\
\text { ciudadanía } \\
\text { democrática }\end{array}$ & $\begin{array}{l}\text { Respuestas simples. Se } \\
\text { entiende la Historia } \\
\text { como mero } \\
\text { pasatiempo y no se } \\
\text { relaciona la Historia } \\
\text { con la acción como } \\
\text { ciudadano. }\end{array}$ & $\begin{array}{l}\text { Se establece una } \\
\text { relación entre } \\
\text { ciudadanía e Historia y } \\
\text { se es consciente de la } \\
\text { utilidad práctica de } \\
\text { ésta, aunque de forma } \\
\text { tímida. }\end{array}$ & $\begin{array}{l}\text { Se analiza } \\
\text { ampliamente cómo la } \\
\text { Historia le ha } \\
\text { permitido formarse } \\
\text { como ciudadano, } \\
\text { observándose cierto } \\
\text { compromiso con el } \\
\text { ejercicio de la } \\
\text { ciudadanía activa. }\end{array}$ \\
\hline $\begin{array}{l}\text { Variable 2: Manejo de } \\
\text { la causalidad histórica }\end{array}$ & $\begin{array}{l}\text { Ausencia o escasez de } \\
\text { causas para justificar } \\
\text { las relaciones entre } \\
\text { hechos presentes y } \\
\text { pasados. }\end{array}$ & $\begin{array}{l}\text { Se es capaz de } \\
\text { establecer relaciones } \\
\text { de causalidad, aunque } \\
\text { de manera limitada. }\end{array}$ & $\begin{array}{l}\text { Se es capaz de hallar } \\
\text { nexos de causalidad } \\
\text { complejos. }\end{array}$ \\
\hline $\begin{array}{l}\text { Variable 3: Capacidad } \\
\text { de posicionamiento } \\
\text { crítico sobre } \\
\text { cuestiones } \\
\text { sociopolíticas } \\
\text { vinculadas a la Historia }\end{array}$ & $\begin{array}{l}\text { Ausencia de } \\
\text { posicionamiento } \\
\text { crítico o incapacidad } \\
\text { de emitir elementos de } \\
\text { juicio suficientes sobre } \\
\text { las problemáticas de } \\
\text { actualidad propuestas. }\end{array}$ & $\begin{array}{l}\text { Se es capaz de } \\
\text { posicionarse } \\
\text { críticamente, aunque } \\
\text { en base a ideas poco } \\
\text { elaboradas. }\end{array}$ & $\begin{array}{l}\text { Se es capaz de } \\
\text { elaborar respuestas } \\
\text { con un grado de } \\
\text { elaboración complejo } \\
\text { en las que se observa } \\
\text { tanto un } \\
\text { posicionamiento sobre } \\
\text { ciertos temas como un } \\
\text { cuestionamiento } \\
\text { crítico de los mismos. }\end{array}$ \\
\hline
\end{tabular}

\section{Resultados y discusión}

Los resultados se presentan por cada variable y describen los niveles de las concepciones de los participantes. Cada nivel se ilustra con ejemplos concretos de unidades de información ${ }^{1}$.

\footnotetext{
${ }^{1}$ De cara a la exposición de las respuestas de los estudiantes en este apartado de resultados, hemos optado por enumerarlas, de tal manera que cuando entre paréntesis indiquemos, por ejemplo, "S5", nos estaremos refiriendo a las respuestas que ha dado el sujeto número cinco.
} 


\section{Variable 1. Valoración de la utilidad de la Historia en relación con el ejercicio de la ciudadanía democrática}

Como se puede ver en la gráfica 1, un 44\% de las respuestas señalan la Historia como un mero pasatiempo y no encuentran una relación directa con el ejercicio de su papel como ciudadanos. Para algunos estudiantes, su utilidad está relacionada con la superación de las Pruebas de Acceso a la Universidad, lo cual pone de relieve el papel central del examen en la práctica escolar (GarcíaMerino, Urionabarrenetxea y Bañales-Mallo, 2016, p. 5):

"No, no me ha ayudado como ciudadano de un sistema democrático" (S7).

Además, los estudiantes suelen recurrir a tópicos como "la Historia se estudia para no repetir los hechos del pasado", sin proporcionar una mayor justificación. Destacan en sus respuestas la confusión de conceptos, como puede verse en el siguiente ejemplo:

"Sí, me ha ayudado, gracias a esta asignatura sé lo que es una democracia, qué diferencias existen con la república o la dictadura y qué cosas buenas y malas han ocurrido durante este periodo, lo cual me sirve para saber si quiero volver a repetir estas circunstancias (si quiero volver a una dictadura o a una república)" (S18).

El nivel dos recoge un 39\% de las respuestas, y aunque las argumentaciones van complejizándose, todavía se recurren a ciertos lugares comunes. Sin embargo, en este nivel ya se vislumbra la importancia que ha tenido el sujeto político "pueblo" en la conquista de ciertos derechos. Asimismo, son varias las respuestas que han sabido valorar la importancia del sistema democrático en el que viven y lo difícil que ha sido llegar hasta él, pudiendo establecer relaciones entre los fenómenos históricos y el ejercicio de la ciudadanía democrática:

"El saber que el hombre que no conoce su historia está condenado a repetirla. Todas las monarquías absolutas, dictaduras y golpes de estado están condenados a fracasar en algún momento por censurar y arrebatar la libertad del pueblo. Donde hay descontentos siempre va a haber revueltas para levantar la voz contra aquello que les oprima" (S14).

El nivel tres recoge sólo un 17\% de las respuestas, sin embargo, estos estudiantes no solo realizan un análisis complejo de los hechos históricos en relación con la propia formación ciudadana, sino también manifiestan un compromiso con la participación dentro de un sistema democrático:

"[Me ha ayudado] claramente [...] sobre todo en los últimos temas, que me han servido porque al cumplir ahora los 18 años he sabido en todo momento cómo participar en las elecciones y cómo se forma el gobierno, así como [entender] el derecho de la mujer [...], haciendo que miles y miles de chicas en mayor cantidad se echen a la calle para manifestarse" (S1).

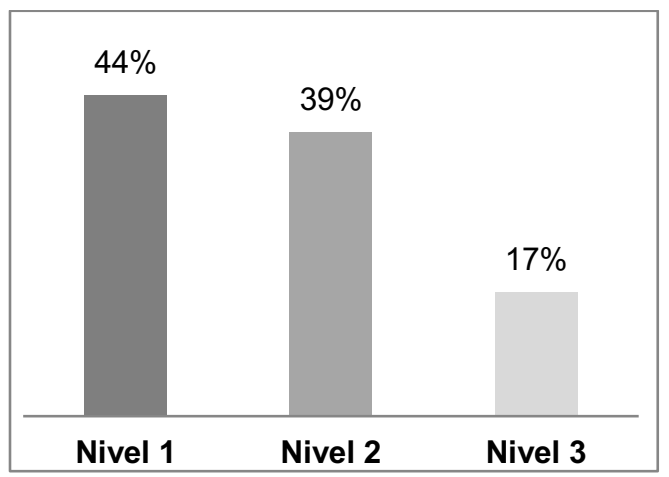

Gráfica 1. Porcentaje de alumnos distribuidos por niveles de la variable 1 


\section{Variable 2. Manejo de la causalidad histórica}

Los resultados de esta variable también ponen de manifiesto que la mayoría de las concepciones de los estudiantes se encuentran en niveles de formulación simple para los problemas planteados, tal y como puede verse en la gráfica 2.

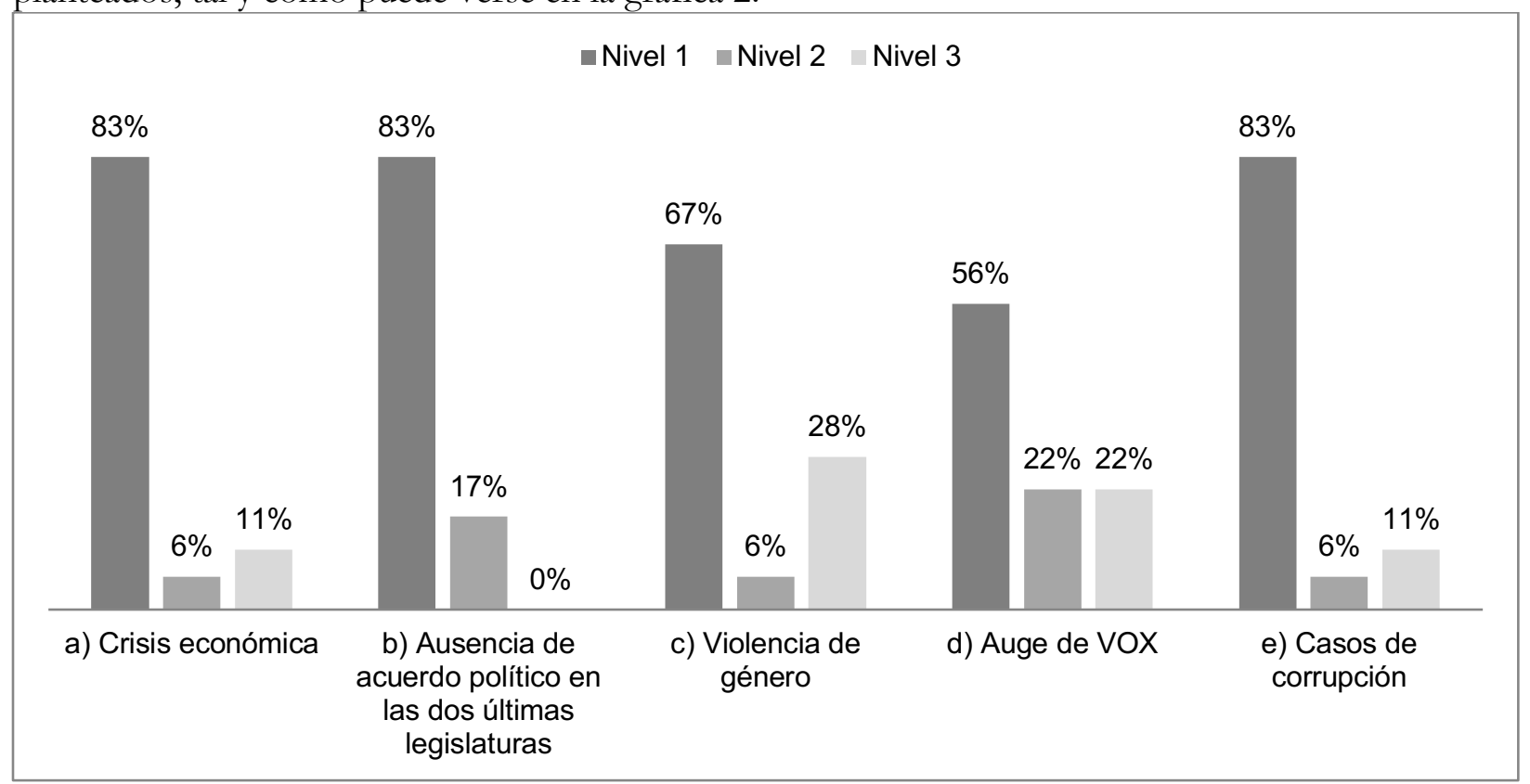

Gráfica 2. Porcentaje de alumnos distribuidos por niveles de la variable 2

En relación a las causas de la crisis económica, las respuestas categorizadas señalan que, tanto en el sistema educativo como en la sociedad en general, se tienden a separar la Economía y la Historia, concibiendo los problemas económicos como un problema de gestión. No se aprecia la existencia de intereses diversos detrás de las crisis económicas ni que los acontecimientos históricos sucedidos puedan explicarlas, cuestiones que entroncan con la propia concepción que el neoliberalismo maneja de la economía. Además, estas respuestas son reflejo de la escasa importancia que tiene la ciencia económica a la hora de explicar la Historia:

"No [existe ningún nexo con algún tema de Historia], yo simplemente pienso que es una mala gestión administrativa" (S10).

Algo muy similar sucede cuando los estudiantes deben establecer relaciones entre la Historia y la ausencia de acuerdo político en las dos últimas legislaturas españolas. La mayoría de las respuestas señalan una especie de eterna incapacidad de los distintos grupos políticos para lograr el entendimiento y a la polarización política, similar a lo vivido durante la Guerra Civil. Dejando a un lado el hecho de que las argumentaciones no ahonden sobremanera en las razones que pudieran explicar la falta de acuerdo político en la España de hoy, las respuestas ubicadas en este nivel constituyen un fiel reflejo de algo que ya mencionábamos anteriormente: el reparto equitativo de culpas y responsabilidades de la Guerra Civil que ha predominado en la mayoría de interpretaciones y manuales de Historia:

"La incapacidad de acuerdo entre los principales grupos políticos es un rasgo que demuestra la división de los ciudadanos; tal y como se encontraba España durante la Segunda República y la Guerra Civil”'(S2).

En cuanto a la cuestión acerca de la violencia de género, las respuestas en este nivel uno llegan a negar el papel de la Historia o de la política en la explicación y resolución del problema de la 
violencia machista. Incluso, y hasta cierto punto, las explicaciones poseen un cierto componente biologicista, achacando el problema de la violencia de género no a cualquier causa de tipo social, sino a las condiciones orgánicas de los individuos:

"No, eso es cosa de enfermos, no tiene nada que ver con la política" (S10).

Por otro lado, en lo que se refiere al auge del partido político VOX, la mayoría de las argumentaciones hacen referencia a que es consecuencia de la polarización social, y no a cuestiones de otro tipo:

"Es consecuencia de la polarización que se está dando en el ámbito político al igual que la que sucedió antes de la Guerra Civil” (S8).

Finalmente, también han sido escasas las explicaciones a las posibles causas de la corrupción política, señalando que siempre va a existir:

"[...] siempre ha existido corrupción y la impunidad de estos casos por conveniencia" (S6).

Las respuestas de los estudiantes ubicadas en el nivel dos señalan que, pese a que son capaces de establecer relaciones de causalidad, estas son limitadas. Así, aunque vinculan la crisis económica con la inestabilidad política de un país, una guerra, la caída de un gobierno o a un proceso inflacionista, sus argumentaciones han sido realizadas de forma general, no focalizándose en la crisis económica de 2008:

"Siempre que hay alguna inestabilidad en un país suele acarrear una crisis económica. La caída de un gobierno, una guerra que acabe con las subsistencias y la inflación son ejemplos que conllevan a la crisis" (S14).

En lo que concierne a la falta de acuerdo político en España, todas las respuestas clasificadas en este nivel han relacionado la falta de consenso con la pervivencia de la idea de las dos Españas, haciendo referencia incluso al periodo de la Segunda República como similar al actual:

"Eso guarda una relación con las posturas e ideologías de cada partido, es decir, al igual que en la Segunda República, la falta de consenso da lugar a una sociedad dividida y la creación de conflictos" (S17).

En lo relativo a la causalidad del problema social de la violencia de género, se destaca que posee una cierta reminiscencia del franquismo:

“[Está relacionada] con la mentalidad conservadora del franquismo [...] aunque la violencia de género proceda de mucho antes debido a la visión que se ha tenido de la mujer a lo largo de la Historia." (S5).

Las respuestas relativas al auge del partido VOX señalan que se debe a una pervivencia de los valores y de la ideología franquista en el seno de nuestra sociedad:

"El auge de Vox viene dado por el amor hacia una España unitaria y en el fondo (no tan profundo) por la pervivencia de ideologías franquistas” (S6).

Por último, y como explicación de este nivel a los casos de corrupción, las respuestas señalan que se deben a una supuesta e histórica pasividad y conformidad con la corrupción por parte de la población:

"[...] se debe a que venimos arrastrando diferentes problemas como mostrar conformidad con la corrupción, o mejor dicho pasividad [...]” (S5).

$\mathrm{El}$ nivel tres de esta variable es, como puede verse en la figura 2, minoritario con respecto al resto. Así, en lo concerniente a la crisis económica de 2008, los estudiantes son capaces de señalar que es responsabilidad de sectores sociales dominantes, como la banca, o incluso que es consecuencia del fin de la dictadura y el inicio del periodo democrático, por la pervivencia de muchos de los grupos que ostentaban el poder en la estructura económica actual:

"Desde la Segunda República, los sectores de las clases altas [...] o la banca han incidido en el mantenimiento de sus privilegios[...]" (S5). 
En cuanto a la cuestión de la violencia de género, un grupo importante de respuestas establecen vínculos entre las situaciones de violencia machista de hoy y el rol de la mujer ama de casa, madre y esposa que la dictadura fomentó durante sus cuarenta años de pervivencia:

"La mujer siempre ha sido vista como inferior al hombre, sobre todo en los partidos de derecha y ultraderecha, y ha costado muchas luchas, manifestaciones y esfuerzos mostrar que las mujeres estamos igualmente capacitadas para realizar cualquier trabajo, y no solo mantener una familia y una casa bajo la protección de un hombre, como exponía el franquismo con naturalidad" (S14).

$\mathrm{El}$ hecho de que esta haya sido una de las cuestiones a las que los estudiantes han contestado con más argumentos y de manera más extensa da buena cuenta de la importancia que hoy día tiene el movimiento feminista en el contexto actual, sobre todo entre los más jóvenes.

En cuanto a la irrupción de VOX, un $22 \%$ de las respuestas manifiestan que se vincula con la herencia que el franquismo y su ideología ha dejado en la España actual. Incluso algunos casos han sido capaces de, indirectamente, asociar el crecimiento de VOX a cuestiones relacionadas con la memoria histórica:

"El auge de VOX es debido a aquellas personas que no conocen su historia y no ven que un partido conservador y de ultraderecha no es admisible en un país que quiere evolucionar. VOX es eso, involución, al igual que lo fue la Falange y Francisco Franco. No podemos consentir esa vuelta atrás en el tiempo y volver a un gobierno que promueva una ideología basada en el odio a los colectivos minoritarios como es el colectivo LGTBIQ+, que además es racista y machista con lemas como: "Ni machismo ni feminismo, igualdad", porque demuestran ignorancia hacia lo que realmente significa el feminismo" (S14).

Declaraciones de este tipo ponen de manifiesto la necesidad de poner en valor la memoria histórica para mantener los sistemas democráticos y para formar ciudadanía participativa y conscientes de las causas y consecuencias que todo acto humano tiene.

En cuanto a la corrupción política, las respuestas no recogen la relación de este fenómeno con los temas del franquismo o la Guerra Civil, sino con cuestiones más concretas como el escándalo del estraperlo:

"En esta asignatura hemos podido ver que desde el caso del Estraperlo ha habido casos de corrupción en ambos sentidos: la izquierda y la derecha. El poder salir impune de un delito así solo tiene una causa posible: el dinero y los chantajes" (S14).

\section{Variable 3. Capacidad de posicionamiento crítico sobre cuestiones sociopolíticas vinculadas a la Historia}

Los resultados para esta variable son notablemente distintos atendiendo a la temática, por lo que hemos optado por realizar una presentación y discusión por cada pregunta. 


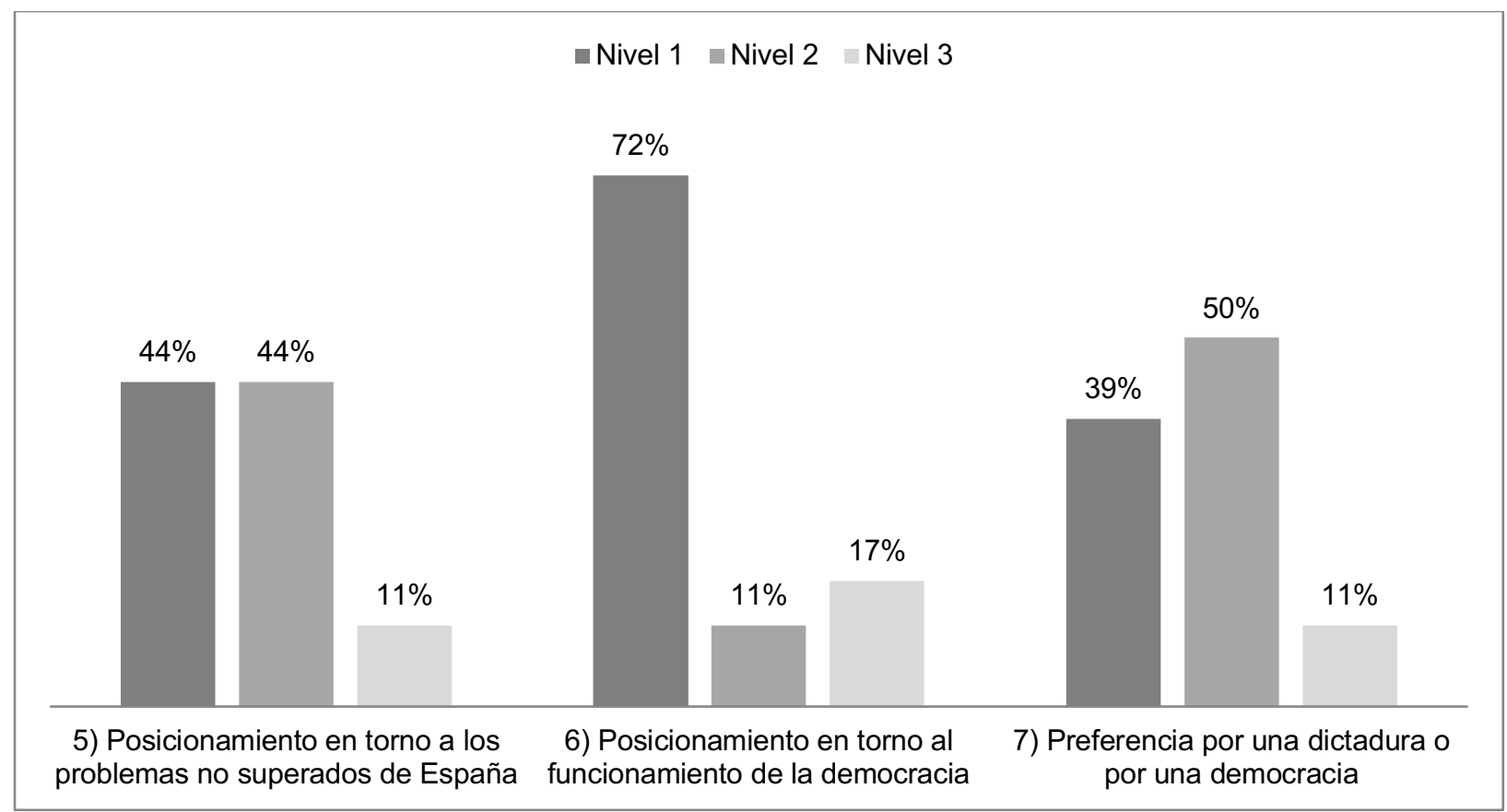

Gráfica 3. Porcentaje de alumnos distribuidos por niveles de la variable 3

Así pues, en lo que respecta a la pregunta cinco, un 44\% de los estudiantes se sitúa en el nivel uno pues aun siendo capaces de posicionarse ligeramente con respecto a problemáticas actuales de España como pudieran ser la independencia de Cataluña o la violencia machista, no posee elementos de juicio ni criterios suficientes para tener una postura crítica con respecto a éstas:

"Sí, claramente, el tema de Cataluña viene de mucho tiempo atrás" (S4).

"Sí, hay problemas sociales que perduran como es la violencia de género" (S9).

En el nivel dos encontramos a un $44 \%$ de los estudiantes, apreciándose un posicionamiento crítico, aunque con ideas poco elaboradas:

"Creo que la Transición en España no dejó las líneas claras. Fue una estrategia exitosa, ya que consiguió la estabilidad, pero hoy día sigue habiendo una brecha entre "bandos" que en los últimos años ha provocado que se polarice la política, a mi parecer, como por ejemplo con el auge de la ultraderecha" (S16).

Finalmente, las declaraciones de los alumnos ubicados en el nivel tres recogen referencias claras a problemáticas actuales tan relevantes como la exhumación de Francisco Franco:

"Las heridas que se produjeron antes y después de la Guerra Civil están abiertas, se ha intentado simplemente tapar todos esos años posteriores de represión y falta de libertad, pero no se ha debido hacer un buen trabajo, dado que hoy día hay diferencias de opiniones sobre la exhumación de Franco o la ley de Memoria Histórica” (S17).

En relación a la pregunta seis se han obtenido una mayoría de respuestas $-72 \%$ - en el nivel uno. En estos casos, los estudiantes no son capaces de presentar argumentos sobre el funcionamiento de la democracia y los que dan, son simples:

"Mal, funciona mal. No creo que guarde relación con nada de la historia [...]. Tampoco se hace nada para remediarlo desde arriba, ya que a los gobiernos les interesa esta situación [...]" (S10).

Aparte de la escasa presencia de argumentaciones críticas, las respuestas categorizadas en este nivel uno dan buena cuenta de dos fenómenos: por un lado, para gran parte de los estudiantes el funcionamiento de la democracia no está tan relacionado con su acción como ciudadanos, sino con 
aquello que hagan los partidos políticos; por otra parte, es consecuencia del descrédito actual que posee la política en la sociedad, así como reflejo del papel pasivo con el que, en numerosas ocasiones, la ciudadanía se ve a sí misma a la hora de ejercer su participación política.

Las respuestas ubicadas en el nivel dos $-11 \%$, , otorgan mayor protagonismo a la participación del pueblo como sujeto importante a la hora de mejorar el sistema democrático. Asimismo, son capaces de relacionar el funcionamiento de la democracia con problemáticas como el papel del Senado o el control político de los medios de comunicación:

"Ha de modernizarse. Instituciones como el Senado son ridículas con la creación de las autonomías [...]. Yo creo que los partidos tienen demasiado control sobre los medios de comunicación, pudiendo provocar una falsa democracia" (S16).

Se han categorizado un 17\% de las respuestas en el nivel tres, donde los estudiantes son capaces de asociar el período republicano vivido en el primer tercio del siglo XX a una democracia plena, destacando sus avances, o incluso cuestionando el funcionamiento de ésta, comparándola con el período de la Restauración ocurrido a finales del siglo XIX:

"La democracia española no funciona mal, pero siempre se pueden hacer mejoras, formas de enriquecer el país de una manera justa sin perjudicar a los de siempre: las clases medias y bajas. Quizá con la Constitución de 1931, en la que hubo grandes avances como el reconocimiento del voto femenino y el sufragio universal[...]" (S14). "Hasta hace unos años la democracia española funcionaba con un bipartidismo y turnismo político que recordaba a la Restauración, pero poco a poco se han ido introduciendo nuevos partidos. Es una democracia que intenta que el sistema no se corrompa, pero desafortunadamente hay muchos casos de acciones ilícitas por parte de nuestros gobernantes" (S17).

En lo que se refiere a la pregunta siete aunque ningún estudiante prefiere un sistema autoritario de gobierno, sí que encontramos un 37\% de respuestas que han asociado la dictadura con estabilidad, desarrollo económico y orden. Ello da buena cuenta de que sigue perviviendo la idea de que un régimen democrático puede ser más ineficaz económicamente que uno dictatorial, suponiendo además el primero cierto caos e inestabilidad:

"Sí y no. Todo depende de los planes reformistas del dictador. Sin embargo, todo tiene un precio, y en el caso de las dictaduras es muy caro, que es la represión a las libertades. Sin represión "no hay dictadura". Sin embargo, hay ocasiones en las que no vendría mal un poco de orden, pero si ese orden conlleva lo que conlleva, claramente no es preferible. Es complicado" (S6).

El 50\% de respuestas que se ubican en el nivel dos para esta pregunta rechazan de manera tajante cualquier gobierno autoritario, asociando la palabra dictadura a conceptos como "represión", "censura", "falta de libertades y de derechos" o "paso atrás":

"No pienso que una dictadura sea un sistema viable, ya que ello implica la represión de la mayor parte de la sociedad y otros aspectos como la censura o la falta de libertades. Opino que la población tiene el derecho a elegir sobre su propia vida e ideales[...]" (S2).

Por último, aquellas respuestas ubicadas en el tercer nivel -11\%- han vinculado la democracia y la consecución de derechos a la lucha del pueblo:

"No. Nunca el poder de una sola figura puede ser algo bueno [...]. Todos tenemos los mismos derechos y hacer que todos tengamos voz y podamos aportar nuestra opinión sobre cómo queremos llevar el país es mejor que obedecer sin protestar a una persona que cree que acortando la libertad de los ciudadanos puede gobernar con facilidad y construir un país moderno y evolucionado" (S14). 


\section{Conclusiones}

Si nos atenemos a la pregunta que ha dado pie a esta investigación - ¿qué tipo de ciudadano sale de las aulas al terminar Bachillerato? - los resultados muestran que, en relación a nuestra primera variable, en una parte nada desdeñable de nuestro grupo de estudiantes predomina la denominada por García Pérez (2012) cultura de la superficialidad", basada en interpretaciones en torno a lugares comunes, no produciéndose una relación adecuada entre los conocimientos adquiridos en Historia de España y su desempeño como ciudadanos de una democracia. Sin embargo, en otro porcentaje importante sí que hemos apreciado una valoración más compleja de la utilidad de la Historia en relación con su formación ciudadana.

En cuanto a nuestro segundo objetivo (por el que queríamos comprobar si los alumnos manejaban la causalidad histórica), la inmensa mayoría ha mostrado dificultades para vincular los hechos históricos del temario de Historia de España referidos a la Segunda República, la Guerra Civil y el franquismo con problemáticas políticas actuales. Además de ello, sus respuestas incluso llegaban a negar, en muchos casos, que la Historia pudiera llegar a guardar algún tipo de relación con acontecimientos de tal calibre como la crisis económica. Ello da buena cuenta, hasta cierto punto, del tipo de paradigma educativo que prima en numerosas ocasiones en nuestras escuelas y en las Ciencias Sociales en general, teniendo éste un claro componente neoliberal o utilitario (Durán, 2012; Gimeno, 2010) que no es más que un claro reflejo del pensamiento dominante (De Alba y García Pérez, 2008) del que hablábamos en la fundamentación teórica.

En referencia a nuestro tercer objetivo, mediante el que queríamos determinar si los estudiantes poseían los elementos de juicio necesarios que les permitieran pronunciarse de manera crítica en relación a ciertos temas de actualidad ligados a la materia de Historia de España, nos es posible afirmar que -a excepción del posicionamiento en torno a la cuestión del adecuado funcionamiento de la democracia española- la clase se halla dividida. Así pues, ha habido una abrumadora mayoría de jóvenes que han sido incapaces de expresar un punto de vista argumentado sobre el modo en que creen que funciona nuestra democracia. Sin embargo, sí que se ha producido un cuestionamiento más crítico y un rol mayor en el ejercicio de la ciudadanía en lo que concierne al posicionamiento de los estudiantes sobre problemáticas no superadas aún en la España actual y sobre la preferencia por una dictadura o una democracia.

Teniendo en cuenta todo lo que acabamos de exponer y volviendo, pues, a la pregunta de investigación con la que dábamos inicio al presente apartado de conclusiones, queda claro que la formación ciudadana de nuestros estudiantes es mejorable. Algo parecido sugieren otro tipo de estudios que han tratado de investigar la relación entre las concepciones y la formación ciudadana de los estudiantes y la enseñanza de la Historia o el rol que desempeña la escuela (Navarro, 2012; Orellana y Muñoz, 2019), los cuales sostienen que -en general- las instituciones educativas y el proceso de enseñanza-aprendizaje en ellas imperante no contribuye a alcanzar la adecuada formación de una ciudadanía democrática.

Por consiguiente, queda patente que la formación en cuestiones de ciudadanía que poseen los estudiantes requiere un cambio educativo que abogue por un replanteamiento de los contenidos en torno a estos temas que nos ocupan (Delgado, 2015), fomentándose una visión que vincule ciudadanía, historia contemporánea de España y memoria. Ello entronca directamente con la pertinencia "de construir un proceso democrático que de fluidez y renovación curricular" (Rangel, 2015, p. 6), teniendo en cuenta además "la posición de los grupos subordinados" (Díez, 2013, p. 405) en los procesos históricos, abogando por una historia de carácter más social (De la Montaña, 2015; Mattozzi, 2015) y organizando los contenidos en torno a problemáticas sociales relevantes (De-Alba-Fernández y Navarro-Medina, 2013). 


\section{Referencias}

Bejines, V. (2016). Relación entre memoria histórica, educación y comunicación. Revista de Educación de la Universidad de Granada, 23, 253-266.

De Alba Fernández, N. y García Pérez, F. F. (2008). ¿Puede la escuela del siglo XXI educar a ciudadanos y ciudadanas del siglo XXI? Scripta Nova: Revista electrónica de Geografía y Ciencias Sociales, 12(270), 1-14.

De-Alba-Fernández, N. y Navarro-Medina, E. (2013). La educación ciudadana de los jóvenes en España. Un ejemplo de investigación cualitativa. En J. Pagès y A. Santisteban (Eds.), Una mirada al pasado y un proyecto de futuro. Investigación e innovación en didáctica de las ciencias sociales (vol. 1) (pp. 719-726). Barcelona: Universitat Autònoma de Barcelona, Servei de Publicacions.

De la Montaña, J. L. (2015). Didáctica de la Historia, Historiografía y la visibilización de temas, grupos sociales y personas en la enseñanza-aprendizaje de la Historia. En A. M. Hernández Carretero, C. R. García Ruíz, y J. L. De la Montaña (Eds.), Una enseñanza de las Ciencias Sociales para el futuro. Recursos para trabajar la invisibilidad de personas, lugares y temáticas (pp. 907-917). Cáceres: Universidad de Extremadura, Servicio de Publicaciones.

Delgado, E. J. (2015). Educación ciudadana y memoria histórica en la enseñanza de Ciencias Sociales. Enseñanza de las Ciencias Sociales, 14, 97-109.

Delval, J. (2012). Ciudadanía y escuela. El aprendizaje de la participación. En N. De Alba Fernández, F.F. García Pérez y A. Santisteban Fernández (Eds.), Educar para la participación ciudadana en la enseñanza de las Ciencias Sociales (vol. 1) (pp. 37-46). Sevilla: Asociación Universitaria de Profesorado de Didáctica en Ciencias Sociales/Diada Editora.

Díez, E. J. (2013). La memoria histórica en los libros de texto escolares. Revista Complutense de Educación, 25(2), 393-409.

Durán, J. F. (2012). La educación y la esfera pública: del proyecto colectivo a las incertidumbres del individualismo mercantilista y democrático. Nómadas. Revista Crítica de Ciencias Sociales y Jurídicas, 35(3), 41-66.

García Díaz, J. E. (1995). La transición desde un pensamiento simple hacia un pensamiento complejo en la construcción del conocimiento escolar. Investigación en la Escuela, 27, 7-20.

García Pérez, F. F. (2012). Una educación para comprender los problemas del mundo e intervenir críticamente. En C. Cruz Rojo, C. Ibáñez Espinosa y S. Moreno Maestro (Coords.), El traje del emperador: 13 propuestas para desnudar al poder (pp. 259-282). Sevilla: Atrapasueños.

García Pérez, F. F. (2016). Educar en la escuela para afrontar los problemas del mundo. En C. De la Rosa de la Vega (Coord.), Más allá de lo imposible. La dimensión política de los derechos humanos en el siglo XXI (pp. 145-171). Tafalla: Txalaparta,.

García-Merino, J. D., Urionabarrenetxea, S. y Bañales-Mallo, A. (2016). Cambios en metodologías docente y de evaluación: ¿mejoran el rendimiento del alumnado universitario? Revista Electrónica de Investigación Educativa, 18(3), 1-18. Disponible en: http://redie.uabc.mx/redie/article/view/691.

Gimeno, J. (Coord.) (2010). Saberes e incertidumbres sobre el curriculum. Madrid: Editorial Morata.

Giroux, H.A. (2015). Democracia, Educación Superior y el espectro del autoritarismo. Revista Entramados- Educación y Sociedad, 2, 15-27.

López Facal, R. (2002). La enseñanza de la historia más allá del nacionalismo. En C. Forcadell Alvarez y J. J. Carreras Ares (Coords.), Usos públicos de la historia: ponencias del VI Congreso de la Asociación de Historia Contemporánea (pp. 223-225). Zaragoza: Prensas Universitarias de Zaragoza /Marcial Pons

Mattozi, I. (2015). La Historia desde abajo en la historia general escolar. En A. M. Hernández Carretero, C. R. García Ruíz y J. L. De la Montaña Conchiña (Eds.), Una enseñanza de las 
Ciencias Sociales para el futuro. Recursos para trabajar la invisibilidad de personas, lugares y temáticas (pp. 259-268). Cáceres: Universidad de Extremadura, Servicio de Publicaciones.

Mayring, Ph. (2014). Qualitative content analysis. Theoretical foundation, basic procedures and software solution (free download via Social Science Open Access Repository SSOAR) Disponible en: https://nbn-resolving.de/urn:nbn:de:0168-ssoar-395173

Moliner, O., Traver, J. A., Ruiz, M. A. y Segarra, T. (2016). Estrategias que inciden en los procesos de democratización de la escuela. Una aproximación teórica. Revista Electrónica de Investigación Educativa, 18(2), 116-129. Disponible en: http://redie.uabc.mx/redie/article/view/1110.

Navarro, E. (2012). La enseñanza de la Historia y el desarrollo de las competencias ciudadanas. El conocimiento del alumnado al finalizar el Bachillerato. Universidad de Murcia, Murcia. Tesis doctoral inédita.

Navarro-Medina, E. \& De-Alba-Fernández, N. (2018). The Construction of a Citizenship Model Through the Teaching of History. In J.A. Pineda-Alfonso, N. De-Alba-Fernández \& E. Navarro-Medina (Eds.), Handbook of Research on Education for Participative Citizenship and Global Prosperity (pp. 500-516). Hershey (USA): IGI Global.

Orellana, C. y Muñoz, C. (2019). Escuela y Formación ciudadana: Concepciones de ciudadanía, formación ciudadana y del rol de la escuela. Revista Electrónica Interuniversitaria de Formación del Profesorado, 22(2), 137-149.

Padilla, E. (2014). Neoliberalismo y educación. Revista de Lenguas Modernas, 20, 337-370.

Rangel, H. (2015). Una mirada internacional de la construcción curricular. Por un currículo vivo, democrático y deliberativo. Revista Electrónica de Investigación Educativa, 17(1), 1-16. Disponible en: http://redie.uabc.mx/vol17no1/contenido-rangelt.html.

Valls, R. (2009). Historia y memoria escolar. Segunda República, Guerra Civily dictadura franquista en las aulas. Valencia: Publicacions de la Universitat de València.

Zaitegi, N. (2004). Educar para la ciudadanía en el siglo XXI. $8^{\circ}$ Congreso Interuniversitario de Organización de Instituciones Educativas (pp. 703-711). Sevilla: Universidad de Sevilla.

Zambrano, E. L. (2018). Prácticas pedagógicas para el desarrollo de competencias ciudadanas. Revista Electrónica de Investigación Educativa, 20(1), 69-82, https://doi.org/10.24320/redie.2018.20.1.1409

\section{Información sobre los autores}

Autor: José Cano Romero

Email: joscanrom@alum.us.es

Autora: Elisa Navarro-Medina

Institución: Departamento de Didáctica de las Ciencias Experimentales y Sociales. Universidad de Sevilla

Email: enavarro5@us.es

ORCID: http:/ / orcid.org/0000-0001-5523-7097 


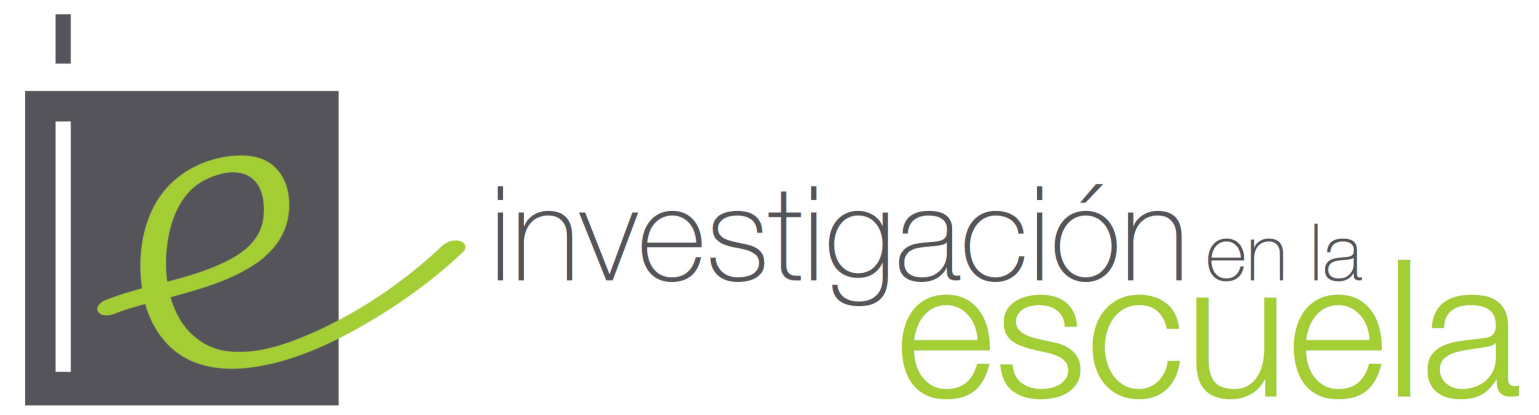

Revista académica evaluada por pares y de acceso abierto

Número 99

30 de diciembre de 2019

ISSN 2443-9991

\section{(c) (i) (2)}

EY Es Esta obra está bajo una licencia Creative Commons. Los/as lectores/as pueden compartir, copiar y redistribuir el material en cualquier medio o formato, así como adaptar, remezclar, transformar y construir a partir del material para cualquier propósito, incluso comercialmente. Para ello, deben de hacerlo bajo los siguientes términos: dando crédito de forma adecuada, brindando un enlace a la licencia e indicando si se han realizado cambios. Si se remezcla, transforma o crea a partir del material, debe distribuir su contribución bajo la misma licencia del original.

Más detalles de la licencia de CreativeCommons se encuentran en https://creativecommons.org/licenses/by-sa/4.0/deed.es

Cualquier otro uso debe ser aprobado en conjunto por el autor/es, o Investigación en la Escuela.

Uิ

Revista Editada por la Universidad de Sevilla. https://editorial.us.es/es/revistainvestigacion-en-la-escuela

Por errores y sugerencias contacte a secretaria@investigacionenlaescuela.es

La revista Investigación en la Escuela desde su origen en 1987 hasta su no 87 (2015) fue editada por Díada Editora. 
